\title{
REFERENCES
}

1. H. Robbins, The asymptotic distribution of the sum of a random number of random variables, Bull. Amer. Math. Soc. vol. 54 (1948) pp. 1151-1162.

2. H. Cramer, Mathematical methods of statistics, Princeton University Press, 1946, p. 365 .

3. Z. W. Birnbaum and M. Sirken, Bias due to non-availability in sampling surveys, Journal of the American Statistical Association vol. 45 (1950) pp. 98-111.

UNIVERSITY OF MICHIGAN

\section{BOUNDS FOR THE COEFFICIENTS OF UNIVALENT FUNCTIONS}

ARYEH DVORETZKY

1. The purpose of this note is to give some estimates for the moduli of the coefficients $a_{n}$ of

$$
w=f(z)=z+a_{2} z^{2}+\cdots+a_{n} z^{n}+\cdots,
$$

assumed regular and univalent in $|z|<1$, in terms of the domain onto which $|z|<1$ is mapped through (1). A typical result, cf. (27), is that if this domain does not cover arbitrarily large circles, then ${ }^{1}$ $a_{n}=O(\log n)$.

Let $W$ be the domain in the $w$-plane onto which $|z|<1$ is mapped through (1) and denote by $A(R)$ the radius of the largest circle with center on $|w|=R$ the whole interior of which is contained in $W$, that is,

$$
A(R)=\max _{|w|=R} \min _{w^{\prime} \notin W}\left|w^{\prime}-w\right| \quad(0 \leqq R<\infty) .
$$

Our aim is to derive upper bounds for $\left|a_{n}\right|$ in terms of $A(R)$.

Always $A(R) \leqq R+1$ while $A(R)=0$ for sufficiently large $R$ if and only if (1) is bounded in $|z|<1$. The function $A(R)$ measures, in some sense, the extension of $W$. This "extension" has, however, little to do with area, in fact whatever the positive function $a(R)$ there exists a function (1) mapping $|z|<1$ on a slit domain and for which $A(R)=o(a(R))$.

Presented to the Society, August 30, 1949; received by the editors March 31, 1949 and, in revised form, July 6, 1949.

1 Throughout the paper, $O$ and $o$ refer to $n \rightarrow \infty$ or $R \rightarrow \infty$ according to which of the variables $n$ or $R$ appears in the formula. 
It is well known that $a_{n}=O(n)$ for all functions (1) while $a_{n}$ $=o\left(n^{-1 / 2}\right)$ for bounded ones. These correspond respectively to no restrictions on $A(R)$ and to $A(R)=0$ for large $R$. As an application of our results we shall find a gradual transition between these extreme cases (cf. [1 $]^{2}$ where some of the results proved here were announced).

Very probably the estimates obtained here do not reflect in most cases even the correct order of magnitude. At any rate it seems that their main interest is in the qualitative, rather than the quantitative, aspect. Therefore we did not push as much as possible the evaluation of the constants involved, and also refrained from giving some rather lengthy refinements (similar to those used by Landau [2] to improve Littlewood's $\left|a_{n}\right|<e n$ ) which would slightly strengthen our results.

2. It is well known that for $|z| \leqq t<1$, (1) satisfies

$$
|f(z)| \leqq M(t)=\frac{t}{(1-t)^{2}} .
$$

If $\beta \notin W$, then

$$
g(z)=\frac{f(z)}{1-f(z) / \beta}=z+\cdots
$$

is also univalent in $|z|<1$. Writing $M$ for $M(t)$ and applying (2) to $g(z)$, we see that if

$$
|\beta|<M
$$

holds, then (1) maps $|z|<t$ on a domain $W_{t}$ exterior to the circle

$$
\left|w-\frac{M^{2} \beta}{M^{2}-|\beta|^{2}}\right|=\frac{M|\beta|^{2}}{M^{2}-|\beta|^{2}} .
$$

Now let $\theta$ be real, $B$ positive, and $b$ satisfy

$$
0<b<1 \text {, }
$$

and assume

$$
0 \leqq R \leqq b M
$$

and

$$
\left|\beta-R e^{i 0}\right|<B \frac{R^{2}}{M}
$$

2 Numbers in brackets refer to the bibliography at the end of the paper. 
Then, if (3) holds, which certainly is the case if

$$
B \leqq \frac{1-b}{b^{2}}
$$

is satisfied, we have

$$
\begin{aligned}
&\left|\frac{M^{2} R e^{i \theta}}{M^{2}-R^{2}}-\frac{M^{2} \beta}{M^{2}-|\beta|^{2}}\right| \\
&=\frac{M^{2}}{M^{2}-|\beta|^{2}}\left|\frac{M^{2}-|\beta|^{2}}{M^{2}-R^{2}} R e^{i \theta}-\beta\right| \\
& \quad=\frac{M^{2}}{M^{2}-|\beta|^{2}}\left|R e^{i \theta}-\beta+\frac{R^{2}-|\beta|^{2}}{M^{2}-R^{2}} R e^{i \theta}\right| \\
& \leqq \frac{M^{2}}{M^{2}-|\beta|^{2}}\left|R e^{i \theta}-\beta\right|\left(1+\frac{R+|\beta|}{M^{2}-R^{2}} R\right) .
\end{aligned}
$$

Thus, if

$$
M\left|R e^{i 0}-\beta\right|\left(M^{2}+R|\beta|\right) \leqq|\beta|^{2}\left(M^{2}-R^{2}\right)
$$

holds, then by (4)

$$
\frac{M^{2}}{M^{2}-R^{2}} R e^{i \theta} \notin W_{t}
$$

We note that, because of (6) and (7), (9) is implied by $b B<1$ and

$$
\left(1+b^{2}+b^{3} B\right) B \leqq\left(1-b^{2}\right)(1-b B)^{2} .
$$

The quadratic equation in $B$ obtained from (11) on replacing the inequality by an equality sign has a unique root in the interval $0<B<1$. Let $B(b)$ be this root. Clearly $B(b) \rightarrow 0$ as $b \rightarrow 1$ and $B(b) \rightarrow 1$ as $b \rightarrow 0$.

It is easily verified that $B=B(b)$ satisfies (8). We have thus from (10) that if

$$
A(R)<B(b) \frac{R^{2}}{M}
$$

then no point of $|w|=M^{2} R /\left(M^{2}-R^{2}\right)$ is contained in $W_{t}$ and thus

$$
W_{t} \subset \frac{M^{2} R}{M^{2}-R^{2}} .
$$

Or, in other words, (5), (6), and (12) imply 


$$
|f(z)|<\frac{M^{2} R}{M^{2}-R^{2}} \leqq \frac{R}{1-b^{2}}
$$

for $|z| \leqq t$. ( $t$ enters through (6) and (13) since $M=M(t)$.)

3. Before applying (13) we go through a reasoning devised by Littlewood [3] and somewhat simplified by Landau [2].

With (1) also

$$
\phi(z)=\left(f\left(z^{2}\right)\right)^{1 / 2}=\sum_{n=1}^{\infty} \alpha_{n} z^{n} \quad\left(\alpha_{1}=1\right)
$$

is regular and univalent in $|z|<1$. If we put

$$
H(t)=\max _{|s|=t}\left|\frac{f(z)}{z}\right| \quad(0 \leqq t<1),
$$

then

$$
|\phi(z)| \leqq(t H(t))^{1 / 2} \quad \text { for }|z| \leqq t^{1 / 2}<1 .
$$

Hence, since (14) is univalent, by evaluating the area

$$
\sum_{n=1}^{\infty} n\left|\alpha_{n}\right|^{2} t^{n} \leqq t H(t)
$$

and on dividing by $t$ and integrating, we get

$$
\sum_{n=1}^{\infty}\left|\alpha_{n}\right|^{2} r^{n} \leqq \int_{0}^{r} H(t) d t \quad(0 \leqq r<1) .
$$

But from (14) we have

$$
\begin{aligned}
\left|a_{n}\right| r^{n}=\left|\sum_{\nu=1}^{2 n-1} \alpha_{\nu} \gamma^{\prime / 2} \alpha_{2 n-\nu} r^{n-\nu / 2}\right| & \leqq \frac{1}{2} \sum_{\nu=1}^{2 n-1}\left(\left|\alpha_{\nu}\right|^{2} r^{\nu}+\left|\alpha_{2 n-\nu}\right|^{2} r^{2 n-\nu}\right) \\
& =\sum_{\nu=1}^{2 n-1}\left|\alpha_{\nu}\right|^{2} r^{\nu} ;
\end{aligned}
$$

thus, by (16), we have for every $0<r<1$

$$
\left|a_{n}\right| \leqq \frac{1}{r^{n}} \int_{0}^{r} H(t) d t .
$$

(Equality can occur only for $n=1$ when $f(z)$ reduces to z.)

4. As a first application we prove the following:

There exists a finite positive $K$ such that 


$$
\limsup _{n=\infty} \frac{\left|a_{n}\right|}{n} \leqq K L
$$

holds for all functions (1) for which

$$
\limsup _{R=\infty} \frac{A(R)}{R} \leqq L .
$$

Proof. Let $b_{0}$ be any value of $b$ satisfying (5), put $B_{0}=B\left(b_{0}\right)$, and choose $L_{0}$ with

$$
0<L_{0}<b_{0} B_{0} .
$$

$A(R)$ is a continuous function of $R$ satisfying $A(0)>0$ and $A(R)$ $\leqq R+1$. Therefore for every $0<t<1$ the equation in $R$

$$
A(R)=B_{0} R^{2} \frac{(1-t)^{2}}{t}
$$

has positive finite solutions. These solutions clearly form a closed set and we denote by $R_{t}=R_{t}\left(f, b_{0}\right)$ the smallest among them. From (13) we have:

If

$$
R_{t} \leqq \frac{b_{0} t}{(1-t)^{2}}
$$

then

$$
\max _{|z| \leqq t}|f(z)| \leqq \frac{R_{t}}{1-b_{0}^{2}} .
$$

Note that if $A(R)$ is replaced in (21) by a larger function, the effect is to increase $R_{t}$.

Let now $L$ satisfy $0 \leqq L<L_{0}$ and take $L^{\prime}$ in the interval $L<L^{\prime}<L_{0}$. Then, using the remark just made, we have for large $R$ (thus necessarily for $t>t_{0}$ with suitably fixed $t_{0}<1$ )

$$
R_{t}<\frac{L^{\prime} t}{B_{0}(1-t)^{2}} .
$$

Because of (20), (22) - and thus also (23) -is satisfied. From (17), (15), (2), (23), and (24) we obtain

$$
\left|a_{n}\right| r^{n} \leqq \int_{0}^{t_{0}} \frac{d t}{(1-t)^{2}}+\frac{L^{\prime}}{B_{0}\left(1-b_{0}^{2}\right)} \int_{0}^{r} \frac{d t}{(1-t)^{2}} ;
$$


integrating and taking $r=1-1 / n$ we have

$$
\left|a_{n}\right|<O(1)+\frac{L^{\prime} e n}{B_{0}\left(1-b_{0}^{2}\right)},
$$

or, since $L^{\prime}$ may be taken as near $L$ as we please,

$$
\limsup _{n=\infty} \frac{\left|a_{n}\right|}{n} \leqq \frac{L e}{B_{0}\left(1-b_{0}^{2}\right)} .
$$

On the other hand we always have according to Littlewood [2] (by an application of (2) and (17) with $r=1-1 / n)\left|a_{n}\right|<e n$. Therefore

$$
K=\max \left(\frac{e}{B_{0}\left(1-b_{0}^{2}\right)}, \frac{e}{L_{0}}\right)
$$

satisfies (18) as required, q.e.d.

5. Next we proceed to show how our results allow the gradual passage from $a_{n}=O(n)$ in the general case to $a_{n}=o\left(n^{-1 / 2}\right)$ in the bounded one:

Let

$$
A(R)=O\left(R^{r}\right)
$$

then we have

$$
\begin{aligned}
& a_{n}=O\left(n^{\gamma /(2-\gamma)}\right) \quad \text { for } 0<\gamma \leqq 1 \text {, } \\
& a_{n}=O(\log n) \quad \text { for } \gamma=0 \text {, } \\
& a_{n}=O(1) \quad \text { for }-2 \leqq \gamma<0 \text {, } \\
& a_{n}=O\left(n^{-1 / 2+2 /(2-\gamma)}\right) \quad \text { for } \gamma<-2 \text {. }
\end{aligned}
$$

Proof. $\gamma=1$ need not be considered and in all other cases $R_{t}$ automatically satisfies (22) (indeed a stronger statement was established in $\$ 4)$.

From (25) and (21) we have for $t>t_{0}$, with suitable $t_{0}<1$ and corresponding $K_{0}$,

$$
R_{t}<K_{0}(1-t)^{2 /(\gamma-2)} .
$$

Applying (17), with $H(t)$ estimated from (2) for $t \leqq t_{0}$, and from (30) in $t_{0}<t<1$ we get (with constant $K_{1}, \cdots, K_{5}$ )

$$
\left|a_{n}\right| r^{n}<O(1)+K_{1} \int_{0}^{r}(1-t)^{2 /(\gamma-2)} d t .
$$


Thus if $0<\gamma<2$ we have

$$
\left|a_{n}\right| r^{n}<K_{2}(1-r)^{r /(r-2)}
$$

while for $\gamma=0$ we get

$$
\left|a_{n}\right| r^{n}<K_{2} \log \frac{1}{1-r}
$$

For $r=1-1 / n$ these formulas yield (26) and (27).

Similarly for $\gamma<0$, we have $\left|a_{n}\right| r^{n}<K_{8}$ and thus (28).

To obtain (29), we estimate from (13) and (30) the area of $W_{t}$, thus obtaining (for $t>t_{0}$ )

$$
\sum n\left|a_{n}\right|^{2} t^{2 n}<K_{4} R_{t}^{2}<K_{6}(1-t)^{4 /(\gamma-2)} .
$$

This, for $t=1-1 / n$, yields

$$
n\left|a_{n}\right|^{2}=O\left(n^{4 /(2-\gamma)}\right),
$$

and thus (29).

6. As remarked at the beginning of the paper, the methods used can be manipulated to give somewhat better results. The $O$ results of the last section can easily be replaced by results involving constants. In particular if, in the assumption (25), $O$ is replaced by $O$, the same may be done in the conclusions (26), (27), and (29).

Also instead of conditions such as (25) referring to $A(R)$ for all $R$ sufficiently large, it is possible to use conditions on mean values of $A(R)$. Thus (28) holds if $\int_{0}^{1} R_{t} d t$ is finite, and this can be secured by suitable assumptions on the mean value of $A(R)$.

Evidently, instead of $R^{r}$ in (25) we could equally well use other functions, for example $e^{R \gamma}$, and so on.

Finally let it be remarked that the methods of this paper may also be applied to other classes of functions, in particular to functions which are $p$-valent (or $p$-valent in the mean).

\section{BIBLIOGRAPHY}

1. A. Dvoretzky, Les coefficients d'une fonction univalente et le domain etale, C.R. Acad. Sci. Páris vol. 225 (1947) pp. 447-449.

2. E. Landau, Über schlichte Funktionen, Math. Zeit. vol. 30 (1929) pp. 635-638.

3. J. E. Littlewood, On inequalities in the theory of functions, Proc. London Math. Soc. (2) vol. 23 (1925) pp. 481-519.

HEBREW UNIVERSITY AND

INSTITUTE FOR ADVANCEd STUdY 